\title{
Reconsidering the "MR Unsafe" breast tissue expander with magnetic infusion port: A case report and literature review
}

\author{
Rami Dibbs ${ }^{1}$, Bozena Culo², Ravi Tandon ${ }^{3}$, Hugo St. Hilaire ${ }^{3}$, Frank G. Shellock ${ }^{4}$, Frank H. Lau ${ }^{3}$ \\ ${ }^{1}$ School of Medicine, Louisiana State University Health Sciences Center, New Orleans, LA; ${ }^{2}$ Loyola Marymount University, Los Angeles, CA; \\ ${ }^{3}$ Division of Plastic \& Reconstructive Surgery, Louisiana State University Health Sciences Center, New Orleans, LA; ${ }^{4}$ Keck School of Medicine, \\ University of Southern California, Playa Del Rey, CA, USA
}

Breast tissue expanders (TEs) with magnetic infusion ports are labeled "MR Unsafe." Therefore, patients with these implants are typically prevented from undergoing magnetic resonance imaging (MRI). We report a patient with a total submuscular breast TE who inadvertently underwent an MRI exam. She subsequently developed expander exposure, requiring explantation and autologous reconstruction. The safety profile of TEs with magnetic ports and the use of MRI in patients with these implants is surprisingly controversial. Therefore, we present our case report, a systematic literature review, and propose procedural guidelines to help ensure the safety of patients with TEs with magnetic ports that need to undergo MRI exams.

Keywords Breast tissue expander / Breast reconstruction / Magnetic resonance imaging / Safety

Received: 2 Dec $2018 \bullet$ Revised: 10 Jan $2019 \bullet$ Accepted: 19 Feb 2019

pISSN: 2234-6163 • elSSN: 2234-6171 • https://doi.org/10.5999/aps.2018.01431 • Arch Plast Surg 2019;46:375-380
Correspondence: Frank G. Shellock Keck School of Medicine, University of Southern California, 7751 Veragua Drive, Playa Del Rey, CA 90293, USA Tel: +1-310-291-6890 E-mail: frank.shellock@mrisafety.com

\section{INTRODUCTION}

The majority of implant-based breast reconstructions utilize tissue expanders (TEs) in the first stage of reconstruction [1]. Because the magnetic infusion ports incorporated into most TEs presumably interact adversely with the electromagnetic fields used for magnetic resonance imaging (MRI), all such TEs are labeled "MR Unsafe" [2-5]. Therefore, the consensus practice is to use other imaging techniques when diagnostic exams are needed for patient management, such as ultrasound or computed tomography [3-5]. However, for certain indications such as neurological, musculoskeletal, or cardiovascular diagnoses, MRI is the superior diagnostic imaging modality. Recently, we treated a patient who underwent first stage breast reconstruction with
TEs. She subsequently underwent MRI following a traumatic shoulder injury and, shortly thereafter, developed expander exposure and required explantation.

To understand the mechanisms whereby an MRI examination could result in TE issues, we performed a literature search. Surprisingly, the data from multiple ex vivo studies and a large clinical series indicated that MRI may not create serious problems for patients with breast TEs that have magnetic ports if special precautions are taken [3-8]. Notably, there are several implants that incorporate magnetic components which are labeled "MR Conditional," permitting patients with those items to undergo MRI exams including a reflux management system; a magnetically-programmable, spinal distraction system; various programmable cerebral spinal fluid shunt valves; and cochlear im- 
plants [2].

Since there are precedents for patients with magnetic implants (including TEs with magnetic ports) to safely undergo MRI $[2,4,5]$, we propose that the "MR Unsafe" labeling of TEs should be reconsidered because special precautions can be implemented to mitigate risks. We present a systematic literature review, a case report, and proposed procedural guidelines to help ensure the safety of patients with TEs with magnetic ports that need to undergo MRI exams.

A systematic review of available English-language, peer-reviewed literature was conducted using PRISMA guidelines and the following electronic databases to identify pertinent publications beginning at the year 1982 (i.e., the approximate date for the beginning of clinical MRI) and ending in 2018: PubMed/ MEDLINE, Google Scholar, EMBASE, and the Cochrane Database of Systematic Reviews. The literature search was conducted using the following combination of terms: "tissue expander" AND "magnetic resonance imaging” OR "MRI." The articles were individually screened for relevance based on their titles, abstracts, and full texts. Relevant articles were carefully reviewed and are contained within.

\section{CASE}

The Institutional Review Board (IRB) approval number that permitted review of patient records for this case report is \#8755. It should be noted that the IRB approved protocol allowing review of patient records did not require patients to sign a consent form because the information was always utilized in a confidential manner and never identified a specific patient.

A 51-year-old woman with invasive ductal carcinoma of the right breast underwent a right nipple-sparing mastectomy with axillary sentinel lymph node biopsy followed by placement of a breast TE (ARTOURA Breast Tissue Expander, $535 \mathrm{~mL}$; Mentor Worldwide LLC, Irvine, CA, USA) [9]. The TE was implanted in a submuscular pocket comprised of pectoralis major and serratus anterior. After incisional closure, $240 \mathrm{~mL}$ of sterile saline was injected into the TE. The patient's intra- and postoperative courses were unremarkable.

At 8 weeks postoperative, the patient was struck by a motor vehicle while crossing the street. She presented to an emergency department, where physical examination demonstrated "very mild erythema" of the right breast with no signs of injury or point tenderness. Due to right shoulder pain, her primary care physician ordered an MRI. During pre-MRI screening, the patient informed the MRI technologist that she had a breast TE. Despite the presence of that implant, the MRI examination was performed. During MRI, the patient reported an uncomfortable sensation in her right breast and the scan was immediately aborted. The reason for the patient's discomfort could not be determined.

Five days later, the patient presented to the emergency department with right breast pain. On physical exam, the breast had two lesions, $2 \mathrm{~cm}$ in diameter, and associated erythema and swelling. She was diagnosed with cellulitis and was prescribed clindamycin. The cause of the cellulitis was likely associated with the trauma sustained from the traffic accident. The patient presented to her plastic surgeon the following day who also noted right inframammary fold erythema and the small lesions (Fig. 1). The saline in the TE was removed. Despite these maneuvers, the right breast continued to worsen and by 3 weeks post-MRI, the patient had impending TE exposure and required

\section{Fig. 1. Expander loss following vehicular accident and MRI}

(A, B) Clinical photographs showing inframammary fold erythema and blistering of the patient's right breast following motor vehicle collision with the patient as a pedestrian and subsequent magnetic resonance imaging (MRI).
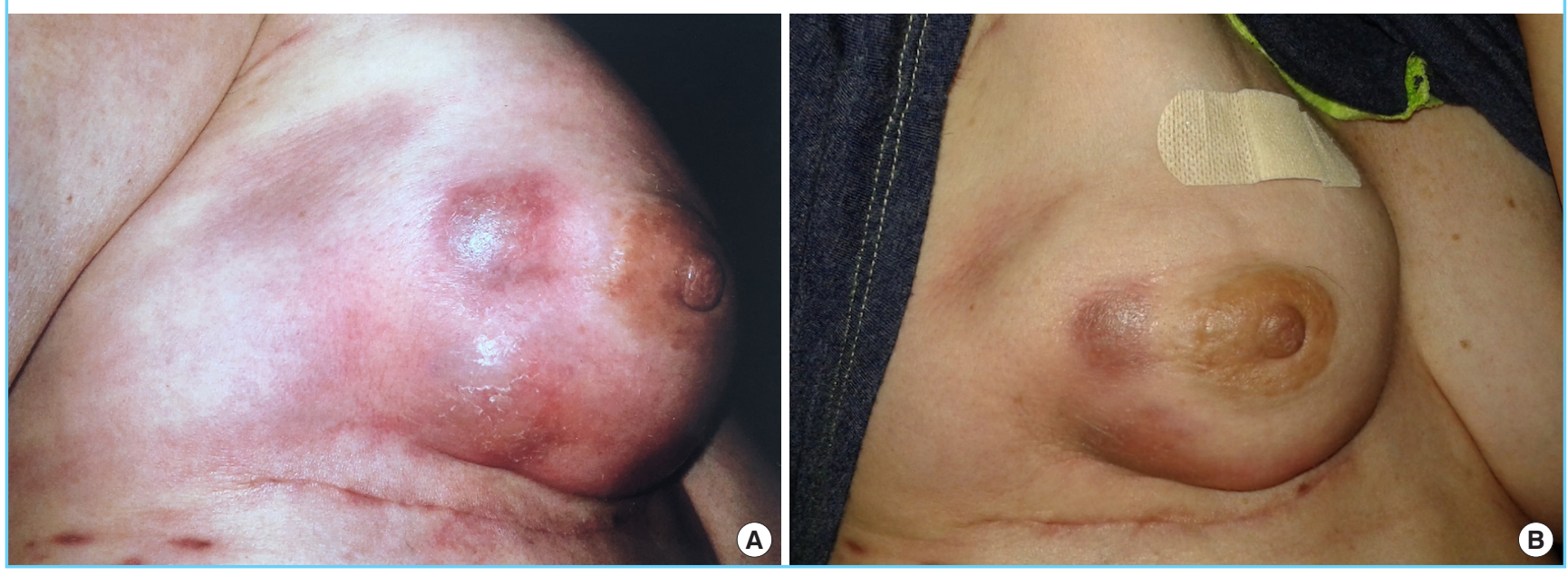


\section{Fig. 2. Acquired breast deformity following tissue expander explantation}

Clinical photographs demonstrating the patient's asymmetric chest after explantation of tissue expander with a magnetic infusion port. (A) Front view. (B) Side view.
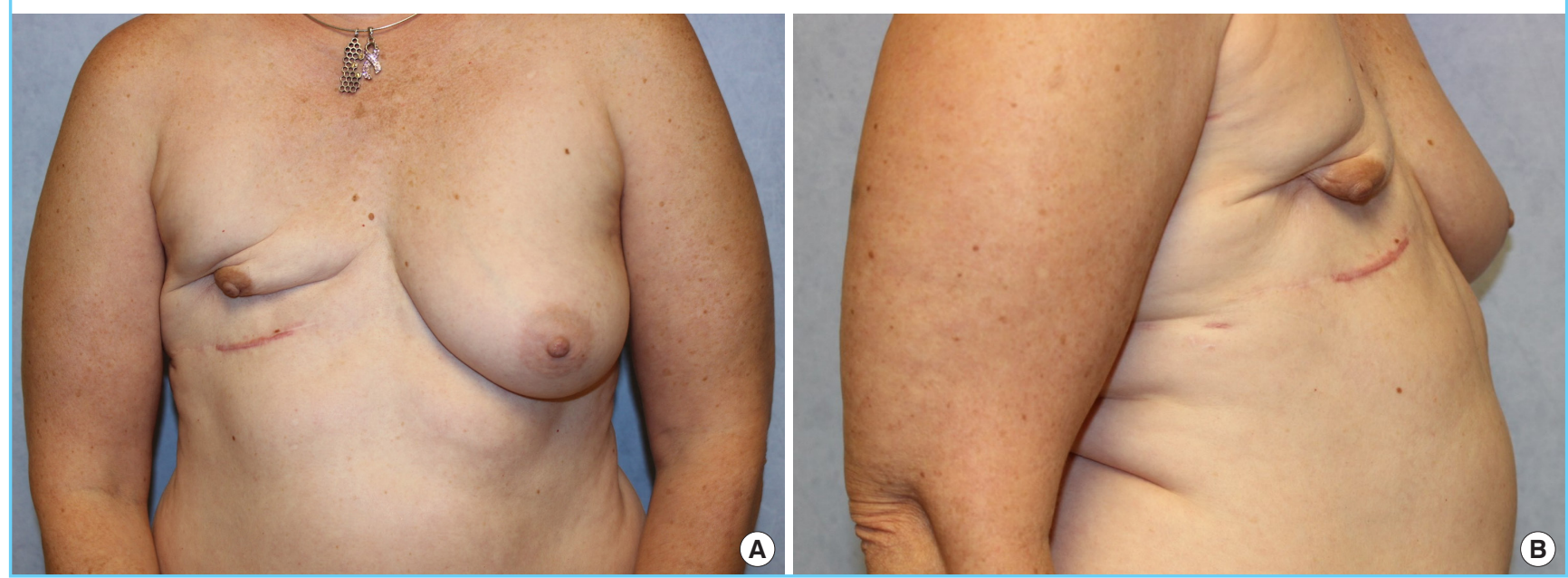

\section{Fig. 3. Delayed breast reconstruction using stacked DIEP flaps}

Patient after undergoing successful right breast reconstruction with stacked deep inferior epigastric artery perforator (DIEP) flaps.

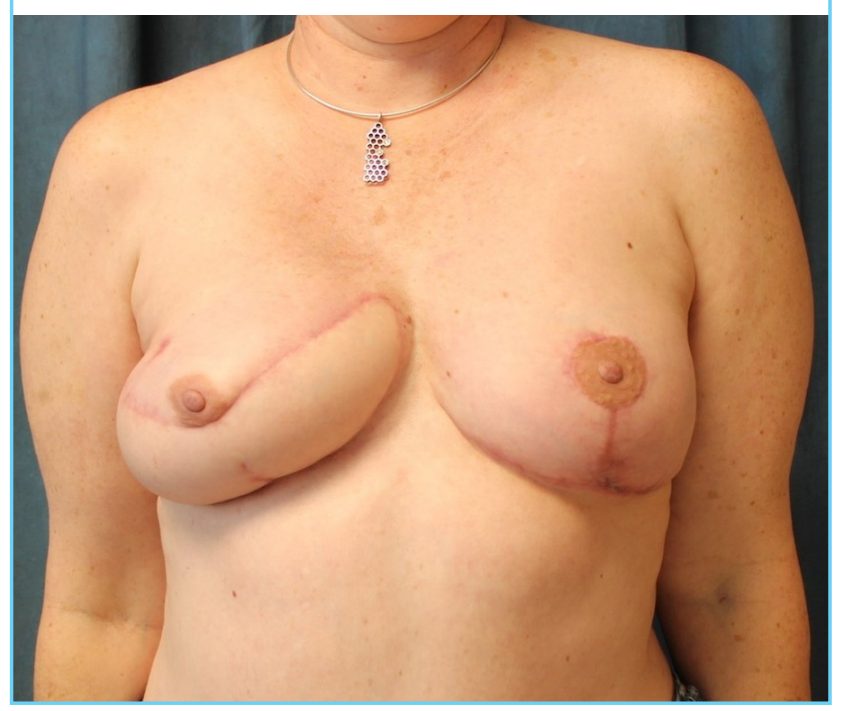

explantation (Fig. 2). Intraoperatively, neither purulence nor fluid collection were present. One year later, the patient successfully underwent breast reconstruction with stacked deep inferior, epigastric artery perforator flaps (Fig. 3). At the time of autologous reconstruction, extensive scar tissue was noted at the mastectomy skin flaps, pectoralis muscle, periosteum, and encasing the internal mammary vessels.

\section{Literature review}

We identified 237 citations in the peer-reviewed literature search (Fig. 4). After eliminating duplicate articles and screen- ing titles, abstracts, and full texts, nine articles were included in the final analysis. The level-of-evidence was relatively low (Table 1). Of the included articles, one was a commentary, two were case reports, and five employed ex vivo testing [3,4,7,8,10-12]. The highest quality study was a retrospective case series involving 71 patients that underwent abdominal/pelvic magnetic resonance angiography (MRA) for autologous breast reconstruction operative planning [5]. Overall, $100 \%$ of ex vivo manuscripts and the large case series indicated minimal risk for TEs with magnetic ports in association with MRI. Notably, only the commentary and two case reports suggested that TEs were unsafe for MRI.

\section{DISCUSSION}

The question of TE safety in association with MRI has been open since at least 1989 [12]. Currently, all TE manufacturers label their devices “MR Unsafe." Interestingly, only two case reports identified in the peer-reviewed literature indicated complications following TE exposure to MRI. Of these, one case described bilateral infusion port dislodgment and the other presented a case of a patient that complained of a "burning sensation" which was neither confirmed nor verified $[10,11]$.

In contrast, several higher level-of-evidence studies indicated that patients with TEs with magnetic ports can be scanned safely using MRI: Nava et al. [3] found that TEs demonstrated minimal heating and had minor attraction to a $1.5 \mathrm{~T}$ static magnetic field and Marano et al. [4] similarly found minimal heating and low magnetic field interactions in three TEs tested under 1.5 and $3 \mathrm{~T}$ MRI conditions. Furthermore, in a retrospective case 


\section{Fig. 4. Schematic of systematic literature review}

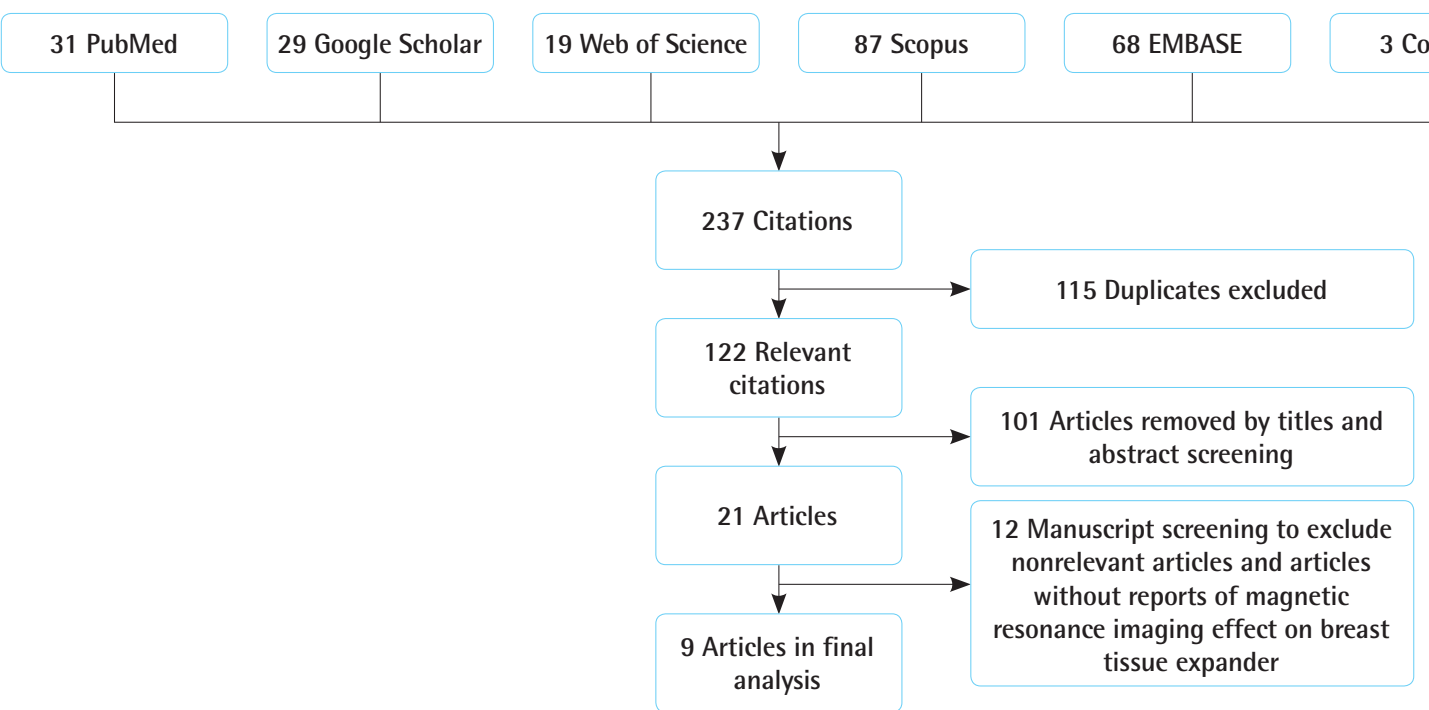

\section{Table 1. MRI and breast tissue expanders with magnetic ports: summary of the review of the literature}

\begin{tabular}{|c|c|c|c|c|}
\hline Author & Year & Study design & No. of patients & Findings/comments \\
\hline Liang et al. [12] & 1989 & Commentary & 0 & $\begin{array}{l}\text { Despite its low mass, the intrinsic magnetic field presents a contra-indicating factor due to the } \\
\text { potential for torque and movement in the presence of the strong magnetic field found in the } \\
\text { clinical MR imagers. }\end{array}$ \\
\hline Fagan et al. [6] & 1995 & Ex vivo testing & 0 & $\begin{array}{l}\text { These results indicate that MR procedures may be performed safely in patients with these } \\
\text { implants. }\end{array}$ \\
\hline Duffy and May [11] & 1995 & Case report & 1 & $\begin{array}{l}\text { Patients with tissue expanders who require MRI scans should be carefully questioned about } \\
\text { localized symptoms in the region of their expander during the scan. }\end{array}$ \\
\hline Zegzula and Lee [10] & 2001 & Case report & 1 & $\begin{array}{l}\text { A chest radiograph was obtained, which demonstrated bilateral dislodgment of the infusion } \\
\text { ports. All patients undergoing tissue expansion with implants that contain integral ports } \\
\text { should be thoroughly warned about the potential hazards of MRI. }\end{array}$ \\
\hline Nava et al. [3] & 2012 & Ex vivo testing & 0 & Under selected conditions, MRI scans can be feasible. \\
\hline Linnemeyer et al. [7] & 2014 & Ex vivo testing & 0 & $\begin{array}{l}\text { A patient with this breast tissue expander with a remote port may safely undergo MRI at 3-T or } \\
\text { less under the conditions used for this investigation. These findings are the first reported at } \\
\text { 3-T for a tissue expander. }\end{array}$ \\
\hline Thimmappa et al. [5] & 2016 & $\begin{array}{l}\text { Retrospective case } \\
\text { series }\end{array}$ & 71 & $\begin{array}{l}\text { MRI/MRA of the abdomen and pelvis in patients with certain breast tissue expanders that have } \\
\text { magnetic ports and are currently labeled "MR Unsafe" can actually be performed safely at } \\
1.5 \mathrm{~T} \text { in the prone position. }\end{array}$ \\
\hline Park et al. [8] & 2017 & Ex vivo testing & 0 & $\begin{array}{l}\text { The increases in SAR are relatively modest and not enough to present a significant heating } \\
\text { risk in tissue. }\end{array}$ \\
\hline Marano et al. [4] & 2017 & Ex vivo testing & 0 & $\begin{array}{l}\text { MRI can be considered in patients with breast tissue expanders when appropriate peri- } \\
\text { procedural choices have been made so that the benefits of undergoing MRI outweigh the } \\
\text { risks. }\end{array}$ \\
\hline
\end{tabular}

series involving 71 patients with 112 TEs, Thimmappa et al. [5] reported that abdominal MRA yielded no reports of magnetic port migration or pain. Subsequent surgeon examination of the peri-TE space during autologous breast reconstruction revealed "no evidence of tissue damage, and there were no operative complications at those sites of breast reconstruction." This group's experience has further increased to more than $250 \mathrm{pa}$ tients without substantial complications (personal communica- tion, Martin R. Prince, M.D., Ph.D., Radiology, Weill Cornell Medical Center, NY, 11/01/18).

In our patient, the right breast TE was several weeks post-implantation and healed. Given the temporal association between her report of the uncomfortable sensation related to the presence of the TE with a magnetic port and her MRI exam, we sought to understand the mechanisms whereby MRI could potentially cause problems. MRI uses a strong static magnetic 


\section{Fig. 5. The ARTOURA Breast Tissue Expander}

Note the position of the magnetic infusion port and that it is covered with nonmetallic, nonconducting material, insulating and isolating it from the patient's tissue. Figure supplied by Mentor Worldwide LLC (Irvine, CA, USA).

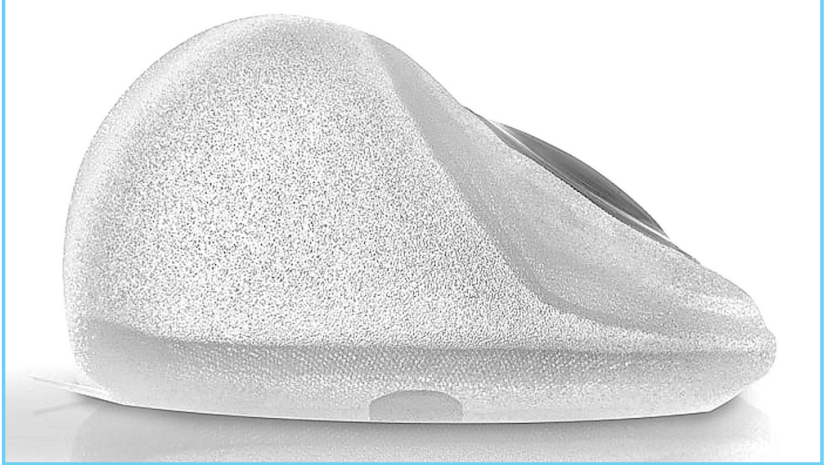

field, time-varying magnetic fields, and radiofrequency (RF) fields, each of which may adversely impact certain implants resulting potential patient injuries or fatalities $[2,13,14]$. With specific reference to MRI-related heating (i.e., the sensation experienced by one patient found in our literature review), RF pulses deposit energy in the patient as a result of the coupling of the electric field and the induction of eddy currents $[2,8,13]$. When a metallic implant is present, the induced currents can generate temperature rises in the surrounding tissues $[2,8,13]$. This type of heating has been investigated for more than three decades. Importantly, these data indicated that only minor temperature changes occur in relatively small metallic objects such as aneurysm clips, prosthetic heart valves, vascular access ports, and TEs with magnetic infusion ports [2-4,6-8]. By comparison, excessive heating can be generated in implants that have an elongated shape, form a closed loop of a relatively large diameter, and that are in direct contact with the patient's tissue $[2,13]$.

A breast TE with a magnetic port is composed of a bladder with an outer shell made from silicone and an internal metallic infusion port to permit saline injections for implant expansion. A permanent magnet is incorporated into the implant to facilitate location of the port. Of note is that the metallic component is isolated and insulated from the patient by a layer of nonmetallic, nonconducting material (Fig. 5). Since the metallic component is small and has no direct contact with the patient's tissues, heating is not a realistic concern.

In conclusion, despite a careful, systematic review, the controversies regarding the safety of a TE with a magnetic port in the MRI setting remain unanswered. Of note is that, during a period of more than three decades, only two cases reported problems associated with TE exposure to MRI $[10,11]$, while a study conducted in a large series of patients where special precautions were taken to mitigate risks revealed no substantial issues [5].

Considering the preponderance of evidence, we recommend that it is time to reconsider whether these implants should be considered "MR Unsafe" and, thus, a strict contraindication for an admittedly vital diagnostic imaging procedure. As part of the practice of medicine, MRI-trained radiologists may elect to override the labeling for implants, even if they are labeled MR Unsafe, by conducting a careful risk versus benefit for individual cases. For example, to date, thousands of patients with "standard” cardiac pacemakers, (which are contraindicated devices) have safely undergone MRI by following a standardized protocol designed to avoid complications [15]. Therefore, in the event that a patient with a TE with a magnetic port needs to undergo MRI for proper clinical management, we propose the following procedural guidelines taking into consideration the clinical experience reported by Thimmappa et al. [5] as well as additional, suitable precautions to prevent possible risks [2,3]. First, the supervising physician (i.e., usually the MRI-trained radiologist) must perform an assessment of the risks associated with the implant versus the benefit of the MRI exam. The theoretical risks or other possible problems include movement of the magnetic port component of the implant, potential MRI-related heating, possible polarity reversal of the magnet, and artifacts in the immediate area of the port $[2,3,5]$. Second, a physician must provide written and verbal informed consent to the patient, explaining the aforementioned possible problems. Third, perform the MRI procedure using a scanner that does not exceed the field strength of $1.5-\mathrm{T}[3,5]$. Fourth, stabilize the TE with a magnetic port by securely wrapping the area with an elastic compression wrap or by other suitable means (e.g., 6" Ace Bandage, elastic chest/rib belt, etc.) [3,5]. Fifth, if practical, place the patient in a prone position to minimize or prevent movement of the magnetic port of the TE $[3,5]$. Sixth, continuously monitor the patient visually and verbally throughout the MRI examination. If the patient reports any unusual sensation, immediately discontinue MRI.

\section{NOTES}

\section{Conflict of interest}

No potential conflict of interest relevant to this article was reported.

\section{Ethical approval}

The study was approved by the Institutional Review Board of Louisiana State University Health Sciences Center (IRB No. 8755 ) and performed in accordance with the principles of the Declaration of Helsinki. 


\section{Patient consent}

The patients provided written informed consent for the publication and the use of her images.

\section{Author contribution}

Study concept and design, data acquisition, drafting of the manuscript, critical revision of the manuscript for important intellectual content, study supervision and approval of the final manuscript: all authors. Data analysis and interpretation, statistical analysis, administrative and technical support: Dibbs $\mathrm{R}$, Culo B, Shellock FG, Lau FH. Administrative support: Tandon R, St. Hilaire H.

\section{ORCID}

Rami Dibbs https://orcid.org/0000-0002-2559-1711

Bozena Culo https://orcid.org/0000-0002-0750-7475

Ravi Tandon https://orcid.org/0000-0002-3109-6557

Hugo St. Hilaire https://orcid.org/0000-0001-7534-907X

Frank G. Shellock https://orcid.org/0000-0002-6823-6031

Frank H. Lau https://orcid.org/0000-0001-7061-4209

\section{REFERENCES}

1. American Society of Plastic Surgeons. Plastic surgery statistics report [Internet]. Arlington Heights, IL: American Society of Plastic Surgeons; c2017 [cited 2018 Nov 11]. https://www.plasticsurgery.org/documents/News/Statistics/2016/plastic-surgery-statistics-full-report-2016.pdf.

2. Shellock FG. Reference manual for magnetic resonance safety, implants, and devices. 2019 Edition. Los Angeles: Biomedical Research Publishing Group; 2019. p. 244-7.

3. Nava MB, Bertoldi S, Forti M, et al. Effects of the magnetic resonance field on breast tissue expanders. Aesthetic Plast Surg 2012;36:901-7.

4. Marano AA, Henderson PW, Prince MR, et al. Effect of MRI on breast tissue expanders and recommendations for safe use. J Plast Reconstr Aesthet Surg 2017;70:1702-7.
5. Thimmappa ND, Prince MR, Colen KL, et al. Breast tissue expanders with magnetic ports: clinical experience at $1.5 \mathrm{~T}$. Plast Reconstr Surg 2016;138:1171-8.

6. Fagan LL, Shellock FG, Brenner RJ, et al. Ex vivo evaluation of ferromagnetism, heating, and artifacts of breast tissue expanders exposed to a 1.5-T MR system. J Magn Reson Imaging 1995;5:614-6.

7. Linnemeyer H, Shellock FG, Ahn CY. In vitro assessment of MRI issues at 3-Tesla for a breast tissue expander with a remote port. Magn Reson Imaging 2014;32:297-302.

8. Park BS, Razjouyan A, Angelone LM, et al. RF Safety evaluation of a breast tissue expander device for MRI: numerical simulation and experiment. IEEE Trans Electromagn Compat 2017;59:1390-9.

9. Mentor Worldwide LLC. Mentor tissue expander product insert data sheet [Internet]. Irvine, CA: Mentor Worldwide LLC [cited 2018 Nov 15]. http://www.mentorwwllc.com/ global-us/SafetyInformation.aspx.

10. Zegzula HD, Lee WP. Infusion port dislodgement of bilateral breast tissue expanders after MRI. Ann Plast Surg 2001; 46:46-8.

11. Duffy FJ Jr, May JW Jr. Tissue expanders and magnetic resonance imaging: the "hot" breast implant. Ann Plast Surg 1995;35:647-9.

12. Liang MD, Narayanan K, Kanal E. Magnetic ports in tissue expanders: a caution for MRI. Magn Reson Imaging 1989; 7 : 541-2.

13. Shellock FG. MRI Issues for implants and devices. In: Shellock FG, Crues JV, Karacozoff AM, editors. MRI bioeffects, safety, and patient management. Los Angeles: Biomedical Research Publishing Group; 2014. p. 375-94.

14. Shellock FG, Woods TO, Crues JV 3rd. MR labeling information for implants and devices: explanation of terminology. Radiology 2009;253:26-30.

15. Nazarian S, Hansford R, Rahsepar AA, et al. Safety of magnetic resonance imaging in patients with cardiac devices. $\mathrm{N}$ Engl J Med 2017;377:2555-64. 